\title{
Current Minimal Invasive Surgery Treatment for Kidney Stones: Bezmialem Experience
}

Muzaffer AKÇAY, Abdulkadir TEPELER, Muhammed TOSUN, İsmail BAŞIBÜYÜK, Fatih ELBİR, Sina KARDAŞ, Tolga AKMAN, Abdullah ARMAGAN, Ali İhsan TAŞÇI

Department of Urology, Bezmialem Vakıf University School of Medicine, İstanbul, Turkey

\section{ABSTRACT}

Objective: We aimed to present the outcomes of patients with symptomatic kidney stones treated with percutaneous nephrolithotomy (PNL) or retrograde intrarenal surgery (RIRS).

Methods: The medical records of patients with symptomatic renal calculi treated with PNL or RIRS between November 2010 and May 2015 were obtained. Demographic characteristics such as age; sex; BMI; stone size and location; and perioperative data including operation, fluoroscopy, and hospitalization time; and success and complication rates were assessed.

Results: Standard (n:336), mini-PNL (n:51), ultramini-PNL (n:37) and microperc (n:47) were performed for 471 renal units. RIRS was the treatment method for 290 renal unites. In the PNL group, the mean patient age was 44.5 (1-83) years and BMI was $26.9 \mathrm{~kg} / \mathrm{m} 2$. The mean stone size was $27.6 \mathrm{~mm}$. In the RIRS group, the mean age and BMI were 47.1 (1-86) years and $25.1 \mathrm{~kg} / \mathrm{m} 2$, respectively. The mean operation, fluoroscopy, and hospitalization times were 70.0 (20-240) $\mathrm{min}, 45.1$ (17-610) s, and 2.4 (1-20) days, respectively, in the PNL group. On the other hand, the mean operation, fluoroscopy, and hospitalization times were 62.5 (40-180) min, 29.8 (0-96) $\mathrm{s}$, and $26.4(12-120) \mathrm{h}$, respectively, in the RIRS group. Whine stone free status was achieved in $88 \%$ in the PNL group; this rate was lower (80\%) in the RIRS group.

Conclusion: Both PNL and RIRS are efficient minimally invasive methods with low morbidity and high success rates for the treatment of symptomatic kidney stone disease.

Keywords: Minimally invasive, PNL, RIRS

\section{Introduction}

In terms of kidney stone incidence, our country is considered to be an endemic area, and it holds an important place in urology practice with an incidence of $11.1 \%$ (1). For the treatment of kidney stones, many methods have been applied up to now, and these methods are extracorporeal shock wave lithotripsy (ESWL), percutaneous nephrolithotomy (PNL), retrograde intrarenal surgery (RIRC), and laparoscopic and open surgery. Owing to technological developments, minimally invasive methods have been mostly implemented in recent years, and the rate of open surgery has fallen below $0.7-4 \%$ (2).

In the European Urology Guide, ESWL and RIRC are generally recommended for the treatment of symptomatic kidney stones smaller than $1 \mathrm{~cm}$, while ESWL or endourologic surgeries (RIRC or PNL) are recommended for stones between 1 and $2 \mathrm{~cm}$, and PNL is primarily recommended for stones larger than $2 \mathrm{~cm}(3)$.

Modern endourological methods have undergone several modifications to reduce complication rates, along with a higher stone-free rate, to improve patient comfort and shorten the length of hospital stays. In order to reduce complication rates by reducing the PNL renal parenchymal tract diameter, as an alternative to standard PNL, micro-PNL, ultra-mini PNL, and mini-PNL techniques have been described (4).

In this study, we aimed to present the results for patients who underwent PNL and RIRC for symptomatic kidney stones between November 2010 and June 2015 in our clinic. 


\section{Methods}

The records of patients who had undergone PNL and RIRC due to symptomatic kidney stones between November 2010 and May 2015 were retrospectively evaluated. Patient consent was preoperatively taken. Demographic data of the patients [age, gender, body mass index (BMI)], average stone size, postoperative stent insertion, surgery and fluoroscopy time, hospital stay, postoperative hematocrit decrease, complication rate, and stone-free rates were evaluated.

Patients' physical examination, routine blood test, urinalysis and culture, direct urinary system graphy (DUSG), renal ultrasonography (USG), intravenous urography, and/or noncontrast computed tomography (CT) scan results were preoperatively evaluated. Patients with positive preoperative urine culture results were treated with appropriate antibiotics, and upon obtaining sterile urine cultures, the patients were operated on. Operations were conducted on patients with sterile urine under antibiotic ( $1 \mathrm{~g}$ of intravenous second-generation cephalosporin) prophylaxis.

\section{Surgical techniques:}

\section{Percutaneous nephrolithotomy:}

Cystoscopy was performed in all patients under general anesthesia in the lithotomy position, and a hydrophilic-tipped guide wire was advanced into the ureter under fluoroscopic guidance. Then, a 6 Fr open-ended ureteral catheter was inserted via the guidewire in order to fill a collecting system with contrast material and was fixed on the urethral catheter. After this procedure, patients were kept into the prone position on a table compatible with C-arm fluoroscopy. Following site clearance and covering the patients, appropriate percutaneous entry via a calyx was performed via an $18 \mathrm{G}$ needle, accompanied by fluoroscopy. For patients who were scheduled for micro-PNL, percutaneous entry was performed via a $16 \mathrm{G}$ needle as it provided a full view. After inserting the guidewire into the collecting system, depending on the operation, the input line was dilated to 8-30 Fr. Then, an Amplatz sheath was placed and after nephroscope in accordance with sheath was placed (6 Fr, $12 \mathrm{Fr}, 17 \mathrm{Fr}, 24 \mathrm{Fr})$, stones were fragmented by pneumatic ultrasonic or laser lithotripsy. Fragments were removed with the help of endoscopic forceps, basket catheters, or by a washing process. The operation was completed with or without nephrostomy tube placement.

\section{Retrograde intrarenal surgery:}

Cystoscopy was performed on all patients in the lithotomy position under general anesthesia, and a hydrophilic-tipped guidewire was advanced into the ureter via fluoroscopy. In order to exclude ureteral pathologies and to perform dilation, with the aid of semi-rigid ureteroscopy (9.5 F, Karl Storz Endoscopy), control ureteroscopy was conducted over the guidewire. Later, under fluoroscopic guidance, an access sheath was advanced over the guidewire to the proximal ureter. Either through the access sheath or through the guidewire for pa- tients for whom a sheath could not be placed, the renal pelvis was reached by means of 7.5-Fr flexible ureterorenoscopy (Karl Storz Endoscopy, flexX2). When it was not possible to reach the kidney due to obstruction, double J (DJ) stents were placed into the ureter, and the procedure was repeated after 2 weeks. The stones were fragmented via a holmium YAG laser until the stones were able to fall spontaneously. Fragments larger than $3 \mathrm{~mm}$ were taken out with a basket for stone analysis. At the end of the operation, for patients with solitary kidney and those with high stone burdens, a 4.8-Fr DJ stent was placed.

After PNL, patients were monitored via hematocrit control due to the bleeding risk. Complications were recorded according to the Clavien system. For all patients, stone-free rates were evaluated with DUSG and USG in the first postoperative month, and patients with non-opaque stones were evaluated with non-contrast CT. Success was defined as being completely stone-free or having residual fragments smaller than $3 \mathrm{~mm}$.

\section{Results}

Of 471 renal units that performed PNL, 47 performed micro-PNL, 37 performed ultra-mini PNL, 51 performed miniPNL, and 336 performed standard PNL; 290 renal units performed RIRC. The average age of the patients in the PNL group was 44.5 (range, 1-83) years; their average BMI was 26.9 (range, $15.3-52.7) \mathrm{kg} / \mathrm{m}^{2}$, and the male/female ratio was 307/164. Operations were performed on the right side in 223 patients, on the left side in 242, and bilaterally in 3. The average stone size was 27.6 (range, 7-130) mm. Among the studied patients, 20 had rotation anomalies, 4 had ectopic pelvic kidneys, 5 had kyphoscoliosis, 2 had solitary kidney, and 1 had a duplicated system.

The average age of the patients who underwent RIRC was 47.1 (range, 1-86) years; their average BMI was 25.1 (range, $15.1-42.7) \mathrm{kg} / \mathrm{m}^{2}$, and the male/female ratio was $158 / 132$. Operations were performed on the right side in 141 patients, on the left side in 127 , and bilaterally in 11 . The average stone size was 13.5 (range, 6-25) mm, and 19 patients were operated on under anticoagulant treatment. Among the patients, 24 had solitary kidney, 6 had rotation anomalies, 6 of horseshoe kidneys, 3 had kyphoscoliosis, 3 had duplicated systems, and 2 had ectopic pelvic kidneys.

The mean durations of PNL and RIRC were 70.0 (range, 20-240) $\mathrm{min}$ and 62.5 (range, 40-180) min, the average durations of fluoroscopy use were 45.1 (range, 17-610) s and 29.8 (range, 4-96) s, and the average hospital stays were 2.4 (range, 1-20) days and 1.1 (range, 0.5-5) days, respectively. After PNL, 416 (88\%) patients had nephrostomy catheters inserted for decompression and drainage. In 15 (5\%) patients who underwent RIRC, an access sheath was not used, and DJ stents were placed in 253 (88\%) patients after the operation. 
Stone-free conditions were achieved in 413 (88\%) patients who underwent PNL and $230(80 \%)$ patients who underwent RIRC. Among the patients who had PNL, 13.1\% developed complications. In terms of complications, 16 patients had prolonged urinary leakage from the nephrostomy tract that required DJ stent installation (Clavien stage-3a), 9 had urinary tract infections (Clavien stage 2), 4 had urosepsis (Clavien stage 4), 3 had urinary extravasation that required drainage (Clavien stage 3a), 5 had hemorrhage that required transfusion (Clavien stage 2), 3 had massive bleeding that required angioembolization (Clavien stage $3 \mathrm{a}$ ), and 4 had pneumothorax or hemothorax that required chest tube insertion (Clavien stage 3a). The complication rate in RIRC was $1 \%$ $(\mathrm{n}=3) ; 2$ patients developed a urinary tract infection (Clavien stage 2) that was controlled by means of antibiotics and 1 patient had urosepsis (Clavien stage 4).

Demographic data, preoperative data, and postoperative success and complications of the patients are shown in Table 1 and Table 2.

\section{Discussion}

The goal of kidney stone treatment is to achieve stone-free conditions with minimal morbidity. Thanks to technological developments and recent minimally invasive surgical methods, not only high success rates have been achieved, but also the length of hospital stay, surgical complications, mortality rates, and workforce loss have decreased. As a result of these recently developed methods, open surgery in the surgical treatment of kidney stones was deserted except in very limited cases, and instead, RIRC and PNL have become routinely applied in modern stone surgery.

The most important handicaps of these minimally invasive methods are that they require a long and difficult learning curve (5). The most important and difficult step of PNL is to perform appropriate percutaneous access from the calyx into the kidney. In the USA, only $11 \%$ of kidney access operations are implemented by urologists (5). In addition, studies have shown that complication rates of kidney access operations that are not implemented by urologists are higher than those that are implemented by urologists (6). The reason for this situation is believed to be that, compared to urologists, radiologists lack surgical experience in terms of unerring caliceal access selection for the most appropriate surgical procedure. In our study, the access procedures in all the cases were conducted by experienced (AT, AA, and TA) urologists.

The success rates of PNL in large series of patients have been reported as being in the range of $72-98 \%(7,8)$. When the factors that affect the success of PNL are taken into consideration, especially the stones' structure, size and location, the patient's previous history of open surgery, and lithotripter type that was used stand out as the most important parameters (8). In our study, the value of $88 \%$ stone-free conditions

\begin{tabular}{|c|c|c|}
\hline & PNL & RIRC \\
\hline n: & 471 & 290 \\
\hline Age & $44.5(1-83)$ & $47.1(1-86)$ \\
\hline BMI $\left(\mathrm{kg} / \mathrm{m}^{2}\right)$ & $\begin{array}{c}26.9 \\
(15.3-52.7)\end{array}$ & $\begin{array}{c}25.1 \\
(15.1-42.7)\end{array}$ \\
\hline \multicolumn{3}{|l|}{ Gender } \\
\hline Male & $307(65)$ & $158(55)$ \\
\hline Female & $164(35)$ & $132(45)$ \\
\hline \multicolumn{3}{|l|}{ Side $(n, \%)$} \\
\hline Right & $223(47)$ & $141(48)$ \\
\hline Left & $242(52)$ & $127(43)$ \\
\hline Bilateral & $3(1)$ & $11(9)$ \\
\hline Stone size $(\mathrm{mm})$ & $27.6(7-130)$ & $13.5(6-25)$ \\
\hline Renal Anomaly & 37 & 44 \\
\hline - Rotation Anomaly & 20 & 6 \\
\hline - Ectopic Pelvic Kidney & 4 & 2 \\
\hline - Horseshoe Kidney & 5 & 6 \\
\hline - Kyphoscoliosis & 5 & 3 \\
\hline - Solitary Kidney & 2 & 24 \\
\hline - Duplicated System & 1 & 3 \\
\hline Use of anticoagulant & - & 19 \\
\hline
\end{tabular}

PNL: percutaneous nephrolithotomy; RIRC: retrograde intrarenal surgery; BMI: body mass index

Owing to the recent developments in endoscopy and lithotripter technology, flexible ureteroscopes with smaller-diameters, better image quality, and increased angulation ability have been used and the RIRC utilization rate in upper urinary tract stone surgery has increased. As a result, particularly in European urology guides, for kidney stones larger than $2 \mathrm{~cm}$, RIRC has become a secondary choice after PNL, whereas for kidney stones smaller than $2 \mathrm{~cm}$, RIRC has become a preferred treatment option. The average success rate in kidney stone treatment with RIRC in one session was found to be $86 \%$ (9). In a study conducted by Grasso, lower calyx stones were grouped as smaller than $1 \mathrm{~cm}$, between 1 and $2 \mathrm{~cm}$, and larger than $2 \mathrm{~cm}$; the success rates were reported as $82 \%, 71 \%$, and $65 \%$, respectively, and it was stated that as the size of the stone increases, the success rate decreases (10). In our study, the success rate was determined as $80 \%$, and the average stone size was calculated as 13.5 $\mathrm{mm}$.

The presence of a bleeding diathesis and a patient being on anticoagulant therapy constitute a contraindication for PNL and ESWL, and thus RIRC is recommended as the most appropriate treatment for these patients (11). In our study, PNL was not applied to any of the patients who were on anticoagulants, while RIRC was administered to 19 patients who were on anticoagulants. 


\section{Table 2. Operational data, success rate, and complications}

\begin{tabular}{|c|c|c|}
\hline & PNL & RIRC \\
\hline n: & 471 & 279 \\
\hline Decease in hematocrit & $3.4(0.1-16.7)$ & $0.7(0.1-5.8)$ \\
\hline Duration of hospitalization & 2.4 (1-20)/day & $1.1(0.5-5) /$ day \\
\hline Duration of nephrostomy (day) & $1.9(0-10) /$ day & - \\
\hline Duration of operation (min) & $70.0(20-240)$ & $62.5(40-180)$ \\
\hline Duration of fluoroscopy (s) & $45.1(17-610)$ & $29.8(4-96)$ \\
\hline Application of nephrostomy & & - \\
\hline Yes & $416(88)$ & - \\
\hline No & $55(12)$ & - \\
\hline \multicolumn{3}{|l|}{ Application of double J stent, \% } \\
\hline Yes & $85(18)$ & $253(88)$ \\
\hline No & $386(82)$ & $37(12)$ \\
\hline \multicolumn{3}{|l|}{ Use of access sheath } \\
\hline Yes & - & $275(95)$ \\
\hline No & - & $15(5)$ \\
\hline \multicolumn{3}{|l|}{ Success (Stone-free) (\%) } \\
\hline Stone free & $413(88)$ & $230(80)$ \\
\hline Rest (>3 mm) & $58(12)$ & $60(20)$ \\
\hline Complication, \% & $62(13.1)$ & $3(1)$ \\
\hline - Pain (medical treatment) & 18 & - \\
\hline - Fever & 9 & 1 \\
\hline -Sepsis & 4 & 2 \\
\hline - Insertion of double J stent & 16 & - \\
\hline - Extravasation & 3 & - \\
\hline -Bleeding & 8 & - \\
\hline Transfusion & 5 & - \\
\hline Embolization & 3 & \\
\hline -Pneumothorax/hemothorax & 4 & \\
\hline $\begin{array}{l}\text { PNL: percutaneous nephr } \\
\text { intrarenal surgery }\end{array}$ & my; $F$ & grade \\
\hline
\end{tabular}

Although PNL is has a high success rate, it must be kept in mind that serious complications might develop during and after surgery. Considering wide series, major complication rates vary between $3.2 \%$ and $6.8 \%(5,12)$. Complications in PNL are classified as major and minor. As postoperative pain is considered to be a minor complication, when this complication is excluded, the rate of other complications is reported to be $9.3 \%$. Hemorrhage is an important cause of morbidity in PNL. The rate of patients with hemorrhage who required transfusion varied from 14 to $23 \%$ in studies $(4,13)$. In our study, the rate of patients with hemorrhage who required transfusion was $1.1 \%$; this low rate was thought to be the result of surgical experience. The rate of patients with hemorrhage who required embolization has been reported as $0.8 \%$ (14). In our study, this rate was found to be $0.6 \%$, which was compatible with that in the literature. In studies that have been conducted, it has been reported that the number and diameter of entries, the stone burden, and the patient's concomitant diseases might be associated with hemorrhage. In recent years, in order to reduce the amount of bleeding, devices with smaller diameters have been used. In our country, ours is one of the first institutions to perform micro-PNL. We performed micro-PNL to 47 patients. Furthermore, recently, 37 patients underwent ultra-mini-PNL through 13-Fr access paths in our clinic.

The probability of the development of serious complications after RIRC is rare. As long as the urinary tract infection is treated with appropriate antibiotics and culture sterility is provided, a serious postoperative infection does not develop (15). In our study, 3 patients (1\%) needed postoperative treatment due to fever and sepsis. The most serious complications after RIRC are ureteral strictures. In the literature, the incidence of ureteral strictures after RIRC is less than $0.5 \%$ (16). Ureteral strictures in the postoperative period were not observed in any of our patients. Generally, patients with hemorrhage during RIRC do not require transfusions. Further, in our study, none of our patients developed hemorrhage that required postoperative transfusion.

\section{Conclusion}

Today, for kidney stones that require treatment, minimally invasive methods such as RIRC and PNL have taken the place of open surgery. As shown in our study, these methods are applied as standard methods for treating kidney stones with high success and low morbidity.

Ethics Committee Approval: Ethics committee approval was not received due to the retrospective nature of this study.

Informed Consent: Informed consent was obtained from patients who participated in this study.

Peer-review: Externally peer-reviewed.

Author Contributions: Concept - A.T.; Design - M.T.; Supervision - A.A.; Funding - A.İ.T.; Materials - İ.B.; Data Collection and/or Processing M.T.; Analysis and/or Interpretation - F.E.; Literature Review - A.T.; Writing - M.T.; Critical Review - A.T.

Conflict of Interest: No conflict of interest was declared by the authors.

Financial Disclosure: The authors declared that this study has received no financial support.

\section{References}

1. Muslumanoglu AY, Binbay M, Yuruk E, Akman T, Tepeler A, Esen T, et al. Updated epidemiologic study of urolithiasis in Turkey. I: Changing characteristics of urolithiasis. Urol Res 2011; 39: 309-14 [CrossRef]

2. Kane CJ, Bolton DM, Stoller ML. Current indications for open stone surgery in an endourology center. Urology 1995; 45: 218-221. [CrossRef]

3. Skolarikos A, Straub M, Knoll T, Sarica K, Seitz C, Petřík A, et al. Metabolic evaluation and recurrence prevention for urinary stone patients: EAU guidelines. Eur Urol 2015; 67: 750-63. [CrossRef]

4. Kukreja R, Desai M, Patel S, Bapat S, Desai M. Factors affecting blood loss during percutaneous Nephrolitotomy: prospective study. J Endourology 2004; 18: 715-22 [CrossRef] 
5. Segura JW, Patterson DE, LeROY AJ, Williams HJ Jr, Barrett DM, Benson RC Jr, May GR, et al. Percutaneous removal of kidney Stones: Review of 1.000 cases. J Urol 1985; 134: 1077-81.

6. Tomaszewski JJ, Ortiz TD, Gayed BA, Smaldone MC, Jackman SV, Averch TD. Renal access by urologist or radiologist during percutaneous nephrolithotomy. J Endourol 2010; 24: 1733-7. [CrossRef]

7. Hasun R, Ryan PC, MArberger M. Percutaneous coagulum nephrolitotripsi: a new approach. Br J Urol 1985; 57: 605-9. [CrossRef]

8. Goldwasser B, Weinerth JL, Carson CC, Dunnick NR. Factors affecting the success rate of percutaneous nephrolitotripsy and incidence of retained fragments. J Urol 1986; 136: 358-60.

9. Wong MY. Flexible ureteroscopy is the ideal choice to manage a $1.5 \mathrm{~cm}$ diamater lower-pole stone. J Endourology 2008; 22: 1845-6. [CrossRef]

10. Grasso M, Ficazzola M. Retrograde ureteropyeloscopy for lower pole caliceal calculi. J Urology 1999; 162: 1904-8. [CrossRef]

11. Papatsoris A, Sarica K. Flexible ureterorenoscopic managment of upper tract pathologies. Urol Res 2012; 40: 639-46. [CrossRef]
12. Wolf SJ, Clayman RV. Percutaneous nephrolitotomy; what is role in 1997. Urol Clin North Am 1997; 24: 42-58.

13. Clayman RV, Mcdougall EM, Nakada SY. Endourology of the upper urinary tract: percutaneous renal nad ureteral procedures. IN:Wals PC, Retik AB, Vaughan EJ, Wein AJ, eds. Campell's urology. Phidelphia: WB Saunders; 1998.p.2789-874.

14. Stoller ML, Wolf JS Jr, St Lezin MA. Estimated blood loss and transfusion rates associated with percutaneous nephrolitotomy. J Urol 1994; 152: $1977-81$.

15. Ague BK, Dahm P, Wu NZ, Preminger GM. Ureteroscopic management of lower pole renal calculi; tecnique of calculus displacement. J Endourology 2001; 15: 835-8. [CrossRef]

16. Watterson JD, Girvan AR, Cook AJ, et all. Safety and efficacy of holmium: YAG lazerlithotrisy in patients with bleeding diastheses. J Urology 2002; 168: 442-5. [CrossRef] 